\title{
Usability Assessment of Neurostim System: A sEMG Activated Neuroprosthesis Remotely Monitorable and Configurable
}

Lucas Malassise Argentim ( $\square$ lucasargentim@gmail.com )

FEl: Centro Universitario da FEl https://orcid.org/0000-0003-1180-236X

Thais Tavares Terranova

FMUSP: Universidade de Sao Paulo Faculdade de Medicina

Linamara Rizzo Battistella

FMUSP: Universidade de Sao Paulo Faculdade de Medicina

Maria Claudia Ferrari Castro

FEl: Centro Universitario da FEI

\section{Research}

Keywords: Electrical Stimulation, Electromyography, User Interface, Stroke, Hemiplegia

Posted Date: December 28th, 2020

DOl: https://doi.org/10.21203/rs.3.rs-127173/v1

License: (c) (i) This work is licensed under a Creative Commons Attribution 4.0 International License. Read Full License 
Lucas M. Argentim ${ }^{1}$, Thai§ T. Terranova ${ }^{2}$, Linamara R. Battistella ${ }^{2}$ and Maria Claudia F. Castro ${ }^{*} \quad 9$

10

${ }^{*}$ Correspondence:

mclaudia@fei.edu.br

${ }^{1}$ Department of Electrical

Engineering, Centro Universitário

FEI, São Bernardo do Campo,

Brazil

Full list of author information is

available at the end of the article
$11-11$

$12-12$

$13-13$

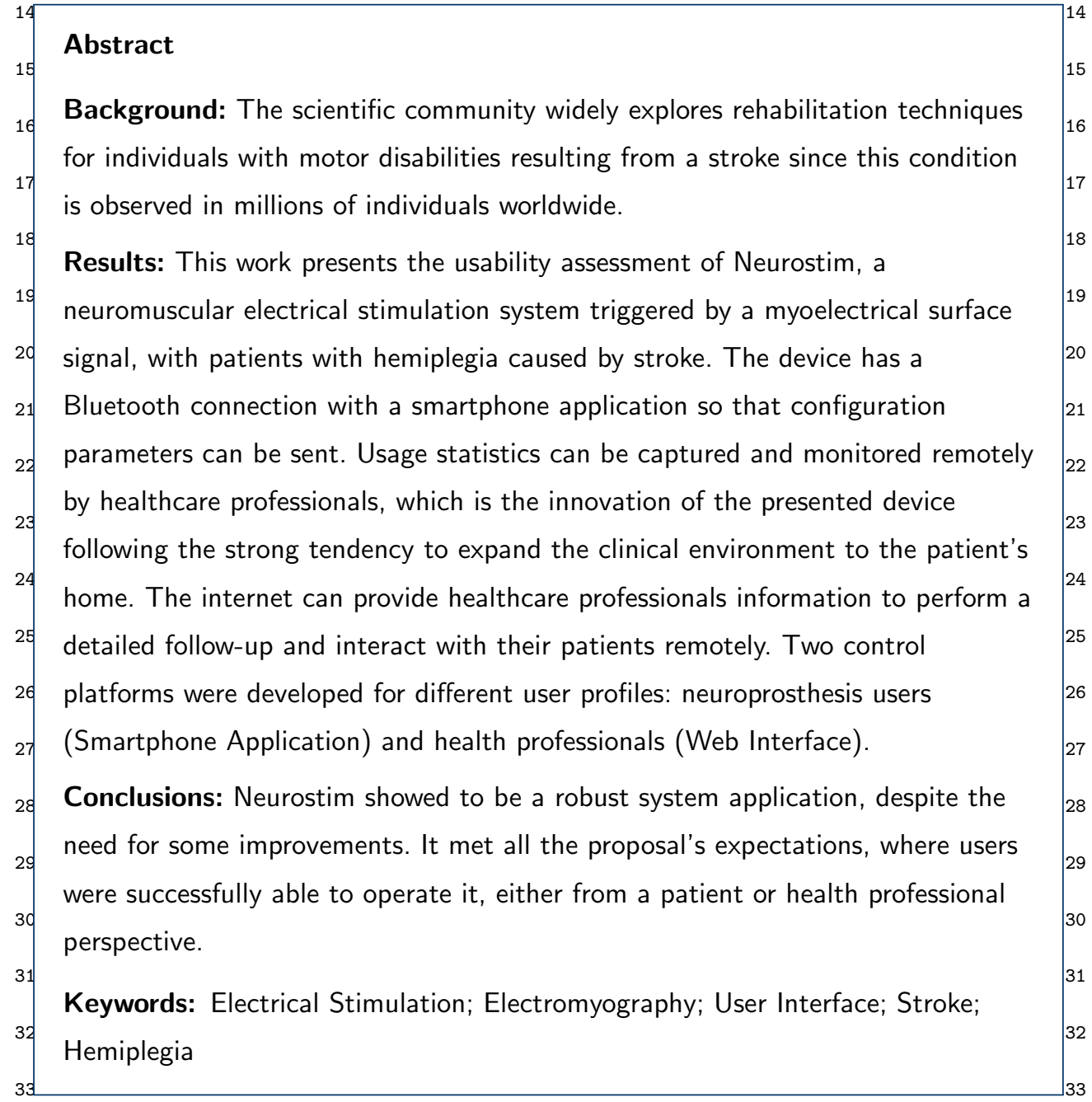


${ }^{1}$ Background

2 (2

Globally, stroke is one of the leading causes of death and long-term disability. It

3 consists of a neurological deficit that occurs when there is a lack of adequate blood ${ }^{3}$

44

flow in a specific area of the brain, either by an obstruction (ischemic, $85 \%$ of

5

cases) or rupture (hemorrhagic, $15 \%$ of cases) of blood vessels. Factors associated

6

with stroke occurrence include individual habits such as inadequate diet, sedentary

lifestyle, smoking, alcoholism, aging, hypertension, and diabetes. Consequently, mo-

8

tor disabilities are commonly observed from brain cells' death in areas responsible for this task $[1-3]$.

11 The damage caused by stroke is directly proportional to its etiology, severity, ${ }_{11}$ ${ }_{12}$ location, patient age, and associated comorbidities. In less aggressive cases, it is ${ }_{12}$ ${ }_{13}$ common to see problems such as temporary weakness of limb muscles. In more ${ }_{13}$ ${ }_{14}$ severe situations, a permanent paralysis on one side of the body or even loss of ${ }_{14}$ ${ }_{15}$ the ability to communicate (expression and interpretation) are typical. In some ${ }_{15}$ ${ }_{16}$ cases, with appropriate treatment, it is possible to recover from stroke completely ${ }_{16}$ ${ }_{17}$ However, more than two-thirds of the survivors will have some sequela or disability, ${ }_{17}$ ${ }_{18}$ compromising the quality of life $[1-3]$.

19 Spasticity is one of the most common impairments after stroke. It is recognized19 ${ }^{20}$ as a phenomenon of velocity-dependent increase in tonic stretch reflexes with exag-20 ${ }^{21}$ gerated tendon jerks caused by an imbalance of the control signals coming from the 21 ${ }^{22}$ Central Nervous System to the muscles. Therefore, there is a muscular hypertonia22 23that prevents voluntary activation for the execution of movements. As an example,23 24the flexor pattern of the elbow, wrist, and fingers is a commonly observed condi-24 25tion in these individuals, that makes it impossible to perform everyday tasks by 25 26themselves, such as feeding, combing hair, brushing teeth, typing in a computer's26 27keyboard, etc. [4, 5].

28 There are numerous reports that the loss of independence is directly associated ${ }^{28}$ ${ }^{29}$ with people's mental health. Here is a considerable decrease in the level of the ${ }^{29}$ ${ }^{30}$ cognitive or emotional quality of life, which can become a picture of depression in ${ }^{30}$ ${ }^{31}$ short time. This condition undoubtedly influences the rehabilitation process, since ${ }^{31}$ ${ }^{32}$ patients undergoing treatment may lose interest in the programs in which they are ${ }^{32}$ ${ }^{33}$ inserted $[3,6,7]$. 
1 Over the years, studies such as [8], [9], and [10] have demonstrated the efficacy ${ }^{1}$

${ }^{2}$ of Neuromuscular Electrical Stimulation (NMES) for motor sequelae from a stroke. ${ }^{2}$

${ }^{3}$ The study presented in [11] showed that patients who use NMES trigged by sur- ${ }^{3}$

${ }^{4}$ face electromyography signal (sEMG) could obtain significant improvement in all ${ }^{4}$

${ }^{5}$ functional tests, and their cortical activation was shifted from the ipsilateral sensori- ${ }^{5}$

${ }^{6}$ motor cortex to the contralateral. This phenomenon could not be noticed in patients ${ }^{6}$

${ }^{7}$ who use only NMES, although they also improved in functional tests. In [9], [12], ${ }^{7}$

${ }^{8}$ and [13], there are pieces of evidence for the positive effects of NMES initiated by ${ }^{8}$

${ }^{9} \mathrm{sEMG}$ in various configurations. However, [14] presented a systematic review and ${ }^{9}$

${ }^{10}$ meta-analysis of this modality's benefits and concluded that these are larger and ${ }^{10}$

${ }^{11}$ evident in chronic cases. In $[15,16]$, the immediate benefits of donning a myoelec- ${ }^{11}$

${ }^{12}$ tric elbow-wrist-hand orthosis in chronic, moderately impaired stroke survivors are ${ }^{12}$

${ }^{13}$ investigated. The use of the system significantly reduced upper limb impairment ${ }^{13}$

${ }^{14}$ and increased the performance of specific functional tasks. 14

15 Given the previous considerations, this work's objective is to evaluate the usabil- ${ }^{15}$

${ }^{16}$ ity of a neuro-prosthetic system, making it possible not only to use it in places ${ }^{16}$

${ }^{17}$ intended for rehabilitation patient's clinic but also at home during the daily life ${ }^{17}$

${ }^{18}$ of these individuals. For this purpose, technologies that allow the capture of sur- ${ }^{18}$

${ }^{19}$ face electromyography (sEMG) and Neuromuscular Electrical Stimulation (NMES) ${ }^{19}$

${ }^{20}$ were used effectively. The sEMG indicates the user's movement intention, while the ${ }^{20}$

${ }^{21}$ NMES helps to complete the movement. The setup of the parameters, activation ${ }^{21}$

${ }^{22}$ intensity, the current intensity of the stimulation, and mode of use are customizable ${ }^{22}$

${ }^{23}$ for each user through an application installed on a smartphone. Sending patient ${ }^{23}$

${ }^{24}$ information to a cloud database allows health professionals to monitor the device's ${ }^{24}$

${ }^{25}$ use and send real-time information back to the patient remotely. 25

26

26

${ }^{27} 1$ Results

27

${ }^{28}$ Table 1 contains the results on the pre and post-test questionnaires for patients ${ }^{28}$

${ }^{29}$ collected respectively at the beginning and the end of the study. In contrast, Table $2^{29}$

${ }^{30}$ shows the results for the pre and post-test questionnaires for health professionals ${ }^{30}$

${ }^{31}$ collected respectively at the beginning and the end of the study. The scale values ${ }^{31}$

used range from 1 to 5 , where 1 stands for the "fully disagree" concept, and $5^{32}$

33 represents the "fully agree" concept. 
$1 \quad * *$ TABLE 1 GOES HERE**

$2 * *$ TABIF 2 GOFS HERE** 2

$3 \quad 3$

4

2 Discussion 5

${ }_{6}$ Between $55 \%$ to $75 \%$ of stroke sufferers will remain with upper limb functional ${ }_{6}$ ${ }_{7}$ limitations after the injury. It will significantly impact the level of dependence on ${ }_{8}$ daily life (ADLs) and their quality of life. In our clinical practice, the non-use of ${ }_{8}$ ${ }_{9}$ the affected upper limb in the ADLs is one of the chief complaints among chronic ${ }_{9}$ ${ }_{10}$ stroke cases, evidencing the need to develop new interventions focused on the upper ${ }_{10}$ ${ }_{11}$ limb functional recovery. At the same time, most recent literature shows that upper $_{11}$ ${ }_{12}$ limb functional recovery can occur even after six months of injury, in the chronic ${ }_{12}$ ${ }_{13}$ phase, due to task-oriented therapeutic interventions performed intensively and ${ }_{13}$ ${ }_{14}$ repetitively [17, 18].

15 NMES was proposed more than 30 years ago. In recent studies, the method has15 16been enriched; it has been combined with a task-oriented approach and trigged16 17through the voluntary effort. In respect of motor relearning, it is vital to ensure17 18personalized treatment, assistance in complex coordinated tasks execution, daily18 19task performance, but with volitional participation of the patient in task execution,19 20avoiding passive mobilization $[18,19]$. In $[15,16]$, using a myoelectric elbow-wrist-20 21hand orthosis significantly reduced upper limb impairment and increased specific21 22functional tasks' performance.

23 Neurostim is a neuroprosthetic device which sEMG trigger the electrical stimulus ${ }^{23}$ ${ }^{24}$ application that can be used both, in the therapeutic and domestic environment, ${ }^{24}$ ${ }^{25}$ under the supervision and according to the guidelines and parameters previously ${ }^{25}$ ${ }^{26}$ provided by the health professional, who can remotely supervise its use, being what ${ }^{26}$ ${ }^{27}$ sets it apart from the others. Thus, a patient can perform therapy more frequently ${ }^{27}$ ${ }^{28}$ and also use it during daily task performance. 28 29 Overall, the first impression of the patients, shown by the pre-test questionnaire ${ }^{29}$ ${ }^{30}$ (Table 1), indicates a good satisfaction level. Question 4 stood out as the furthest ${ }^{30}$ ${ }^{31}$ from the expected perception, with a standard deviation demonstrating different ${ }^{31}$ ${ }^{32}$ opinions according to each patient. The assigned low score refers to the fact that ${ }^{32}$ ${ }^{33}$ the entire assembly of the parts of the neuroprosthesis was done and all instructions ${ }^{33}$ 
${ }^{1}$ given by the Occupational Therapist responsible for the session so that the patient ${ }^{1}$

${ }^{2}$ could use the application.

3 As for the complexity of using the system, expressed by patient P1's scores in ${ }^{3}$ ${ }^{4}$ questions 3 and 8 , it is believed that, as he was the only one who had this opinion and ${ }^{4}$ ${ }^{5}$ was the first to be presented to the study and to attend the sessions, it could have ${ }^{5}$ ${ }^{6}$ been a consequence of the inaugural session and the learning curve of the technical ${ }^{6}$ ${ }^{7}$ aspects of the presentation of the device, which was an activity performed by the ${ }^{7}$ ${ }^{8}$ responsible for this study. Throughout the other meetings, the other patients $\operatorname{did}^{8}$ ${ }^{9}$ not have the same impression of this topic. After the tenth session, it was expected ${ }^{9}$

${ }^{10}$ that the pre-judgments that could have influenced the first evaluation were remedied ${ }^{10}$

${ }^{11}$ and thus obtained more reliable impressions about the user's actual feelings about ${ }^{11}$

${ }^{12}$ the system. 12

13 Observing the results of Table 1 - post-test, it can be observed a decrease in ${ }^{13}$ ${ }^{14}$ standard deviations, indicating that there was a convergence in patient's opinion ${ }^{14}$ ${ }^{15}$ and significant decrease in the mean value for the column that contains the difference ${ }^{15}$

${ }^{16}$ between the assigned scores and the desired value, previously in pre-test being $0.55^{16}$

${ }^{17}$ points and moving to 0.38 points in post-test. This reduction in the mean value ${ }^{17}$

${ }^{18}$ indicates that the assigned scores were closer to the expected value, demonstrating ${ }^{18}$

${ }^{19}$ an increase in familiarity with the system. Patients liked the interface and felt ${ }^{19}$ ${ }^{20}$ comfortable using it.

21 However, question 4 continued to stand out as the furthest from the expected ${ }^{21}$ ${ }^{22}$ perception, and with more patients reiterating the impression that it would take ${ }^{22}$

${ }^{23}$ the help of a technician to use the device. When questioned, the opinion of all who ${ }^{23}$ ${ }^{24}$ expressed themselves negatively revolved around not knowing what value to choose ${ }^{24}$ ${ }^{25}$ for sensitivity for sEMG when it should be adjusted, even though the values are in ${ }^{25}$ ${ }^{26}$ a dimensional escalation. This behavior confirms that the presence of a sensitivity ${ }^{26}$ ${ }^{27}$ selection aid routine would be an essential improvement for future releases. $\quad 27$ ${ }^{28}$ For question 2, there is an improvement of 1-point for patient P2, converging ${ }^{28}$ ${ }^{29}$ to the same opinion of patients $\mathrm{P} 3$ and $\mathrm{P} 4$, and a 2-point worsening for patient ${ }^{29}$ ${ }^{30} \mathrm{P} 1$, which reaffirms his initial perception about finding the system complicated. ${ }^{30}$

${ }^{31}$ When questioned, the patient states that there was technical implications, but $\operatorname{did}^{31}$ ${ }^{32}$ not know what aspects he referred to, preventing him from using the system. This ${ }^{32}$

${ }^{33}$ feelin is 33 feeling is in agreement and also justifies the scores to questions 4 and 10. 
1 For question 6, a 2-point worsening is noted for patient P3. When questioned, this ${ }^{1}$

${ }^{2}$ impression was because the device stopped working at the beginning of the ninth ${ }^{2}$

${ }^{3}$ session, which had to be interrupted and subsequently canceled, so that the repair ${ }^{3}$

${ }^{4}$ could be performed. Undoubtedly, this type of event directly reflects the reliability ${ }^{4}$

${ }^{5}$ that the patient has on the device. ${ }^{5}$

6 6

${ }^{7}$ For the Occupational Therapists, it is evident from Table 2 that the assigned ${ }^{7}$

${ }^{8}$ scores were more distant from the desired values, showing a more reticent and ${ }^{8}$

${ }^{9}$ critical feeling about the system. Small standard deviations show similar opinions ${ }^{9}$

${ }^{10}$ in most of the items evaluated. The perception of Occupational Therapists at the ${ }^{10}$

${ }^{11}$ end of the study also improved, despite remaining more critical. The column's mean ${ }^{11}$

${ }^{12}$ value that contains the difference between the assigned scores and the desired value ${ }^{12}$

${ }^{13}$ was from 1.53 to 1.33 points. This change was caused by the increase in the score ${ }^{13}$

${ }^{14}$ awarded by the OT1 since the OT2 maintained the same scores as in the pre-test ${ }^{14}$

${ }^{15}$ questionnaire. It is interesting to note that none of the Occupational Therapists ${ }^{15}$

${ }^{16}$ attributed the maximum score to the presented statements. These results indeed ${ }^{16}$

${ }^{17}$ indicate that there are still opportunities for improvement. $\quad{ }^{17}$

$18 \quad 18$

19 Although the evaluation was carried out in a controlled environment, where quali- ${ }^{19}$

${ }^{20}$ fied professionals performed the placement of Neurostim, and with a small sample of ${ }^{20}$

${ }^{21}$ users, all of them could interact with the system brought evidence of some potential ${ }^{21}$

${ }^{22}$ improvements before allowing system application in unmonitored situations: $\quad 22$

23

24 - Adjustment of the bracelets that fix the electrodes, integrating them in one 24

25

- Adjustment of the bratets that fix the electrodes, integrating them in one 24 peace, allowing the user to wear it without assistance.

26

- Increased drawing area on the Smartphone screen for dynamic objects, making ${ }^{26}$ it easier to interact with buttons, sliders, etc.

- Confirmation of the end of the procedure before sending data to the server ${ }^{28}$ prevents unexpected stimulation during transmission.

- Adjustment in the scales of the axis values obtained by the sEMG and values ${ }^{30}$ chosen for the sensitivity parameter.

- Confirmation request to end procedure to avoid finishing by an accidental ${ }^{32}$ touch on the screen. 
${ }^{1} 3$ Conlcusion 1

${ }^{2}$ As the main contribution of this work, the literature review showed that a combined ${ }^{2}$

${ }^{3}$ initiative between NMES and sEMG applied to a portable device, with the ability ${ }^{3}$

${ }^{4}$ to send and receive data connecting to a smartphone application, and providing ${ }^{4}$

${ }^{5}$ support tools and remote monitoring for health professionals, had not yet been ${ }^{5}$

${ }^{6}$ proposed. The pioneering of this development will undoubtedly stimulate continuity ${ }^{6}$

${ }^{7}$ in improving the design and development of related tools. Given the benefits that ${ }^{7}$

${ }^{8}$ an integrated solution for low-cost rehabilitation can bring, it is expected that this ${ }^{8}$

${ }^{9}$ platform will become versatile enough to make it a continuous use item, outside of ${ }^{9}$

${ }^{10}$ rehabilitation activities, being incorporated into the patient's day-to-day life until ${ }^{10}$

${ }^{11}$ its use is expendable. 11

12 Neurostim showed to be a reliable system application, despite the need for some ${ }^{12}$

${ }^{13}$ improvements. It met all the proposal's expectations, where users were successfully ${ }^{13}$

${ }^{14}$ able to operate it, either from a patient or health professional perspective. Its design ${ }^{14}$

${ }^{15}$ was versatile enough for the user to feel comfortable wearing it as a physiothera- ${ }^{15}$

${ }^{16}$ peutic element in the rehabilitation center, with intentions of expanding the use to ${ }^{16}$

${ }^{17}$ a practical way outside the clinic to help perform daily tasks that are no longer ${ }^{17}$

${ }^{18}$ possible due to muscle disability. The smartphone application was designed so that ${ }^{18}$

${ }^{19}$ a simple interface allows the user to perform all requested activities with ease, pre- $^{19}$

${ }^{20}$ venting eventual errors during the stimulation procedures and avoiding behaviors ${ }^{20}$

${ }^{21}$ that would not be expected. The web interface was also developed so the health pro- ${ }^{21}$

${ }^{22}$ fessionals can have relevant information about patient's usage and perform detailed ${ }^{22}$

${ }^{23}$ analysis to perform adjustments to maximize effectivity to patients' treatment, all ${ }^{23}$

24 remotely.

24

${ }^{27} 4.1$ Neurostim 27

${ }^{28}$ Neurostim usage aims to rehabilitate the upper limbs (hands and wrists) of patients ${ }^{28}$

${ }^{29}$ with hemiplegia. It is an entirely custom made neuroprosthesis, which relies on the ${ }^{29}$

${ }^{30}$ application of Neuromuscular Electrical Stimulation (NMES) activated by surface ${ }^{30}$

31 . 31

${ }^{32}$ and used in clinical rehabilitation programs. Figure 1 illustrates the device mounted ${ }^{32}$

33 and being used by a user. 


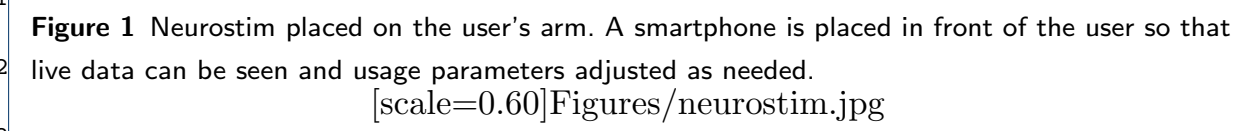

5 The innovation corresponds to the proposal of daily use in all environments (not ${ }_{5}$ ${ }_{6}$ only at the rehabilitation center). For this prospect, two control platforms were 6 ${ }_{7}$ developed: one for operating the neuroprosthesis and another platform for remotely ${ }_{8}$ monitoring its activities, allowing the health professional to consume data from the 8 ${ }_{9}$ device, and to set up parameters of usage, which includes physiotherapy agenda, 9 ${ }_{10}$ flow monitoring, and qualitative analysis.

13The user interface is an Android smartphone application, built using Android Studio13 142.3.3. It is meant to be run in Android versions 4.0 and superior. The application14 15can configure and control the neuroprosthesis with the necessary instructions on15 16the fly. With the electric current application, the extension of wrist and fingers can16 17be achieved when the user's intension of movement is captured through the sEMG17 18circuit.

19 The application can also act as a bi-directional communication channel between ${ }^{19}$ ${ }^{20}$ the healthcare professional and the patient, so setups, adjustments of the usage ${ }^{20}$ ${ }^{21}$ parameters, and information sending about the usage can be made remotely through ${ }^{21}$ 22the internet (either with a Wi-Fi connection or cellular data).

23 Users of this application present motor function disabilities in one side of their ${ }^{23}$

${ }^{24}$ body, commonly causing them to lose the wrist and fingers' movements partially. ${ }^{24}$

${ }^{25}$ The interface was developed considering the placement of buttons and dynamic ${ }^{25}$

${ }^{26}$ objects on the screen to be easily reachable while using just one hand. 26

27 All data is stored locally, considering that the smartphone might not have an ${ }^{27}$

${ }^{28}$ active internet connection by the time the procedure ends; thus, synchronization ${ }^{28}$

${ }^{29}$ cannot happen. With this mechanism, synchronization can be trigger later on when- ${ }^{29}$

${ }^{30}$ ever a stable connection is available. 30

31 Figure 2 shows one of the application screens that contain the live usage data from ${ }^{31}$

${ }^{32}$ a user, where the user can also control the intensity of the electrical stimulation and ${ }^{32}$

33 the sEMG sensibility. 
Figure 2 Smartphone application procedure page, showing live information from the current session to the patient.

3 $[$ scale $=0.5]$ Figures $/$ app.jpg

${ }_{6}$ The web interface for the health professional was developed in HTML5, PHP, and 6

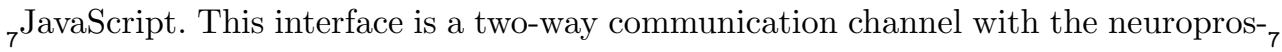
${ }_{8}$ thesis, providing the necessary information to configure its use and an instrument ${ }_{8}$ ${ }_{9}$ of interaction between the patient data and the health professional. By synchro- ${ }_{9}$ ${ }_{10}$ nizing the captured neuroprosthesis data from the respective user's smartphones, ${ }_{10}$ ${ }_{11}$ the healthcare professional can fetch the device's usage history information, so pro- ${ }_{11}$ ${ }_{12}$ gression analysis from a clinical standpoint can be done and remotely perform the ${ }_{12}$ ${ }_{13}$ necessary adjustments, as all the data will be stored in a cloud MySQL database. ${ }_{13}$ 14 The web interface can display a significant amount of information in a graphical $_{14}$ ${ }_{15}$ format, simplifying the data's understanding and providing detailed information as ${ }_{15}$ ${ }_{16}$ needed for the health professional. It also has tools to automatically indicate to the ${ }_{16}$ ${ }_{17}$ health professional if the scheduled session was executed following the previously ${ }_{17}$ ${ }_{18}$ set parameters. Hence, it becomes easier to evaluate the progress and forecast the ${ }_{18}$ ${ }_{19}$ upcoming sessions of each patient.

20 Figure 3 shows one of the application screens that contain the usage data from $\mathrm{a}_{20}$ ${ }_{21}$ patient, received right after the user completed the session.

${ }^{27}$ The clinical usability trials were performed with patients over 18 years old, of both ${ }^{27}$ ${ }^{28}$ genders, with clinical and radiological diagnosis of stroke, motor disabilities in an ${ }^{28}$ ${ }^{29}$ upper limb due to the presence of right or left hemiparesis, who already be in a reha- ${ }^{29}$ ${ }^{30}$ bilitation program using functional electrical stimulation in muscle groups for wrist ${ }^{30}$ ${ }^{31}$ and fingers extension and who already use smartphone as a daily routine activity. ${ }^{31}$ ${ }^{32}$ All the participants were granted in writing of informed consent to participate in the ${ }^{32}$ ${ }^{33}$ study. It is important to note that not only the patients but also the Occupational ${ }^{33}$ 
${ }^{1}$ Therapists (referred to as Health Professionals) were part of this study, showing the ${ }^{1}$

${ }^{2}$ facilities and difficulties found during the assembly of the neuroprosthesis and the ${ }^{2}$

${ }^{3}$ use of the respective interfaces intended for them. ${ }^{3}$

4 The data collection was done with four patients in the rehabilitation process, all ${ }^{4}$

${ }^{5}$ with right spastic hemiplegia, two were male, and two were female, ranging from $19^{5}$

${ }^{6}$ to 46 years old. Initially, the objective was to carry out the study with at least ten ${ }^{6}$

${ }^{7}$ patients. However, the inclusion criteria did not allow such amount in the period ${ }^{7}$

${ }^{8}$ available to carry out the study and to provide proper documentation with the ${ }^{8}$

${ }^{9}$ results. The health professional group had two occupational therapists responsible ${ }^{9}$

${ }^{10}$ for the selected patients' rehabilitation program's regular activities, both female ${ }^{10}$

${ }^{11}$ being 35 and 56 years old. 11

$12 \quad 12$

${ }^{13} 4.5$ Evaluation sessions 13

${ }^{14}$ The procedures' objective was to verify whether the developed interfaces are in- ${ }^{14}$

${ }^{15}$ telligible and accessible to the respective user profiles (patients and health profes- ${ }^{15}$

${ }^{16}$ sionals), the adequacy of communication, and the neuroprosthesis response to the ${ }^{16}$

${ }^{17}$ received commands. This research complied with the tenets of the Declaration of ${ }^{17}$

${ }^{18}$ Helsinki and was approved by the Institutional Review Board at the research ethics ${ }^{18}$

${ }^{19}$ committee of the HCFMUSP, under the number 00240918.2.0000.0068. Informed ${ }^{19}$

${ }^{20}$ consent was obtained from each participant. 20

21 Both patient and health professional were submitted to a presentation session ${ }^{21}$

${ }^{22}$ on the proposed usability study, and one evaluation quiz was completed by each, ${ }^{22}$

${ }^{23}$ according to their personal information and personal characteristics regarding fa- ${ }^{23}$

${ }^{24}$ miliarity with digital devices, technology, frequency of smart devices usage, that ${ }^{24}$

${ }^{25}$ could help understand some of the usability results. Pre and post-test question- ${ }^{25}$

${ }^{26}$ naires about user profile perception of system usage were also applied for posterior ${ }^{26}$

$\begin{array}{ll}27 \\ { }^{27} \text { critical analysis. } & 27\end{array}$

${ }^{28}$ The patients were submitted to 10 sessions, these being once a week, lasting one ${ }^{28}$

${ }^{29}$ hour each. The session's duration considers the placement of the neuroprosthesis ${ }^{29}$

${ }^{30}$ by the therapist, execution of activities requested to both therapist and patient, ${ }^{30}$

${ }^{31}$ disassembly of the neuroprosthesis, and oral evaluation of the group session. Table $3^{31}$

${ }^{32}$ lists the activities requested for each participant. 32

33 **TABLE 3 GOES HERE** 33 
1 In all sessions, patients were recorded using the application that controls the neu- ${ }^{1}$

${ }^{2}$ roprosthesis. In this way, one could monitor what facilities and difficulties each one ${ }^{2}$

${ }^{3}$ had for the given tasks. In the case of Occupational Therapists, the use of the web ${ }^{3}$

${ }^{4}$ interface was monitored by recording directly from the operating system's graphical ${ }^{4}$

${ }^{5}$ environment using Webex Recorder (Cisco) software. Data were also collected to ${ }^{5}$

${ }^{6}$ identify the eventual difficulties of the users while operating the system. 6

$\begin{array}{ll}7 & 7\end{array}$

8Acknowledgements $\quad 8$

Thank you to Centro Universitário FEl, for providing the necessary conditions to conduct this study.

${ }^{9}$ Acknowledgments to FAPESP for funding process 2017/10816-9, which this study is proud to be part of. 9

${ }^{10}$ Funding 10

${ }_{11}$ FAPESP process 2017/10816-9 has funded all costs associated to development and deployment of this project. 11

${ }_{12}$ Abbreviations 12

sEMG - Surface Electomiography

${ }^{13}$ NMES - Neuromuscular Electrical Stimulation $\quad 13$

${ }_{14}$ OT - Occupational Therapist 14

${ }_{15}$ Availability of data and materials

The datasets generated and/or analysed during the current study are not publicly available due to patient

16 confidentiality, but are available from the corresponding author on reasonable request. 16

17Ethics approval and consent to participate $\quad 17$

This research complied with the tenets of the Declaration of Helsinki and was approved by the Institutional Review

${ }^{18}$ Board at the research ethics committee of the HCFMUSP, under the number 00240918.2.0000.0068. Informed

19 consent was obtained from each participant. 19

${ }_{20}$ Competing interests $\quad 20$

The authors declare that they have no competing interests. 21

Consent for publication

${ }^{22}$ Not applicable. $\quad 22$

${ }^{23}$ Authors' contributions $\quad 23$

24 LMA was the engineer and developer of all platforms presented. Dr. TTT selected the participants based on the 24 acceptance criteria for this project and supervised the sessions from a medical standpoint, along with the vast

25 experience of Dr. LRB. Dr. MCFC masterfully coordinated the study and was a major contributor in writing the 25

26 manuscript. All authors read and approved the final manuscript.

Author details

${ }^{27}{ }^{1}$ Department of Electrical Engineering, Centro Universitário FEI, São Bernardo do Campo, Brazil. ${ }^{2}$ Institute of 27 28Physical Medicine and Rehabilitation (IMRea), FMUSP, São Paulo, Brazil. 28

29References 29

1. Tadi, P., Lui, F.: Acute Stroke (Cerebrovascular Accident). StatPearls: StatPearls Publishing, Treasure Island,

30 FL, USA (2020). https://www.ncbi.nlm.nih.gov/books/NBK535369/

31 2. WHO: Neurological disorders: a public health approach. Neurological disorders: public health challenges. WHO 31 Press, Geneva, Switzerland (2006).

32 https://www.who.int/mental_health/publications/neurological_disorders_ph_challenges/en/ 32

33 3. Ramos-Lima, M.J., Brasileiro, I.d.C., Lima, T.L.d., Braga-Neto, P.: Quality of life after stroke: impact of 33 clinical and sociodemographic factors. Clinics 73 (2017). doi:10.6061/clinics/2017/e418 
1 4. Kuo, C.-L., Hu, G.-C.: Post-stroke spasticity: A review of epidemiology, pathophysiology, and treatments.

2 International Journal of Gerontology 12(4), 280-284 (2018). doi:10.1016/j.ijge.2018.05.005

5. Li, S.: Spasticity, motor recovery, and neural plasticity after stroke. Frontiers in Neurology 8, 120 (2017)

3 doi: $10.3389 /$ fneur. 2017.00120

6. Charfi, N., Trabelsi, S., Turki, M., Mâalej Bouali, M., Zouari, L., Dammak, M., Ben Thabet, J., Mhiri, C., Mâalej, M.: Impact of physical disability and concomitant emotional disturbances on post-stroke quality of life.

5 L'Encéphale 43(5), 429-434 (2017). doi:10.1016/j.encep.2016.05.014 (1)

7. Rudberg, A.-S., Berge, E., Gustavsson, A., Näsman, P., Lundström, E.: Long-term health-related quality of life, survival and costs by different levels of functional outcome six months after stroke. European Stroke Journal

7 3(2), 157-164 (2018). doi:10.1177/2396987317753444 7

8. Kralj, A., Ačmović, R., Stanič, U.: Enhancement of hemiplegic patient rehabilitation by means of functional

8 electrical stimulation. Prosthetics and Orthotics International 17(2), 107-114 (1993). 8

9 doi:10.3109/03093649309164365 9

9. Hara, Y.: Neurorehabilitation with new functional electrical stimulation for hemiparetic upper extremity in

10 stroke patients. Journal of Nippon Medical School 75(1), 4-14 (2008). doi:10.1272/jnms.75.4 10

11 10. Lin, Z., Yan, T.: Long-term effectiveness of neuromuscular electrical stimulation for promoting motor recovery 11 of the upper extremity after stroke. Journal of Rehabilitation Medicine 43(6), 506-510 (2011).

12 doi:10.2340/16501977-0807 12

11. Shin, H.K., Cho, S.H., Jeon, H.-S., Lee, Y.-H., Song, J.C., Jang, S.H., Lee, C.-H., Kwon, Y.H.: Cortical effect and functional recovery by the electromyography-triggered neuromuscular stimulation in chronic stroke 13

14 patients. Neuroscience Letters 442(3), 174-179 (2008). doi:10.1016/j.neulet.2008.07.026 14

12. Lee, Y., Cha, Y., Kim, Y., Hwang, S., Chung, Y.: Effect of repetitive wrist extension with

15 electromyography-triggered stimulation after stroke: a preliminary randomized controlled study. Physical 15

16 Therapy Rehabilitation Science 6(3), 127-133 (2017). doi:10.14474/ptrs.2017.6.3.127 16

13. Jonsdottir, J., Thorsen, R., Aprile, I., Galeri, S., Spannocchi, G., Beghi, E., Bianchi, E., Montesano, A.,

17 Ferrarin, M.: Arm rehabilitation in post stroke subjects: A randomized controlled trial on the efficacy of 17 myoelectrically driven fes applied in a task-oriented approach. PLoS One 12((12):e0188642) (2017).

doi:10.1371/journal.pone.0188642

1914. Monte-Silva, K., Piscitelli, D., Norouzi-Gheidari, N., Batalla, M.A.P., Archambault, P., Levin, M.F.:

Electromyogram-related neuromuscular electrical stimulation for restoring wrist and hand movement in poststroke hemiplegia: A systematic review and meta-analysis. Neurorehabilitation and Neural Repair 33(2),

21 96-111 (2019). doi:10.1177/1545968319826053

15. Peters, H.T., Page, S.J., Persch, A.: Giving them a hand: Wearing a myoelectric elbow-wrist-hand orthosis

22 reduces upper extremity impairment in chronic stroke. Archives of Physical Medicine and Rehabilitation 98(9), 22

23 1821-1827 (2017). doi:10.1016/j.apmr.2016.12.016 23

16. Qian, Q., Hu, X., Lai, Q., Ng, S.C., Zheng, Y., Poon, W.: Early stroke rehabilitation of the upper limb assisted

24 with an electromyography-driven neuromuscular electrical stimulation-robotic arm. Frontiers in Neurology 8,24 447 (2017). doi:10.3389/fneur.2017.00447

25 Levin, M.F., Kleim, J.A., Wolf, S.L. What do motor "recovery" and "compensation" mean in patients following 25

26 stroke? Neurorehabilitation and Neural Repair 23(4), 313-319 (2009). doi:10.1177/1545968308328727 26

18. Dobkin, B.H.: Strategies for stroke rehabilitation. The Lancet Neurology 3(9), 528-536 (2004).

27 doi:10.1016/S1474-4422(04)00851-8

28 19. Micera, S., Caleo, M., Chisari, C., Hummel, F.C., Pedrocchi, A.: Advanced neurotechnologies for the 28 29 restoration of motor function. Neuron 105(4), 604-620 (2020). doi:10.1016/j.neuron.2020.01.039

Tables 
3Table 1 Results of the questionnaire for patients

${ }_{14}$ Table 2 Results of the questionnaire for Occupational Therapists

Table 3 Tasks to be executed

Task Executor

Description

T1 OT Mount the neuroprosthesis on the patient

T2 OT

Create / check / modify the patient parameter setting schedule

T3 Patient Perform in-app user authentication

Patient End procedure 


\section{Figures}

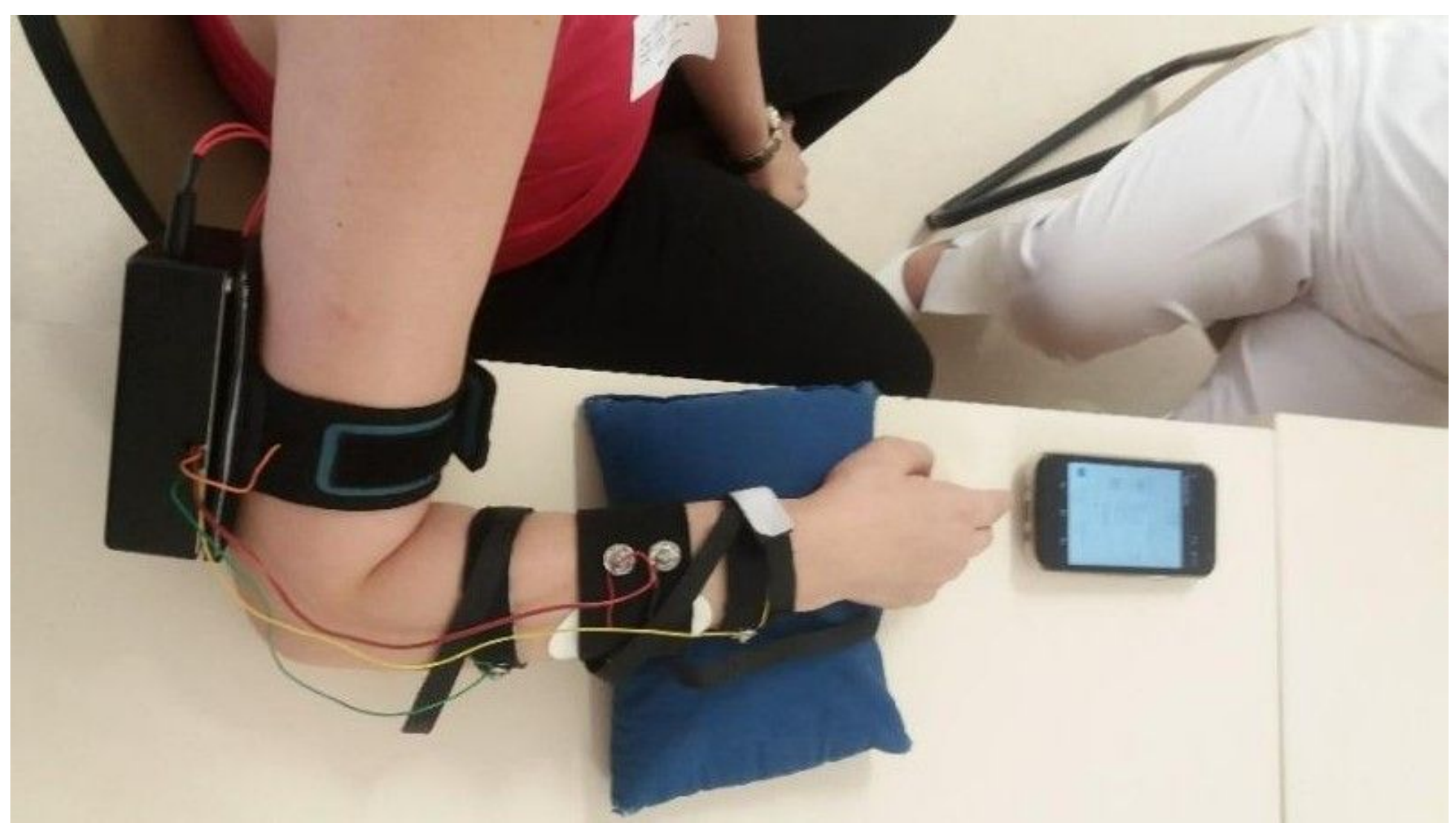

\section{Figure 1}

Neurostim placed on the user's arm. A smartphone is placed in front of the user so that live data can be seen and usage parameters adjusted as needed. 


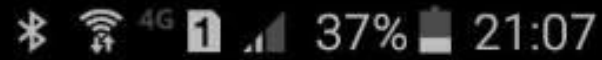

\section{NEUROSTIM}

Current Value

9 Date

Sensibility

Intensity

Time

Schedule

29/10/2017:21:03

50

Repetitions
8

25

*

99
Intensity

Intensity

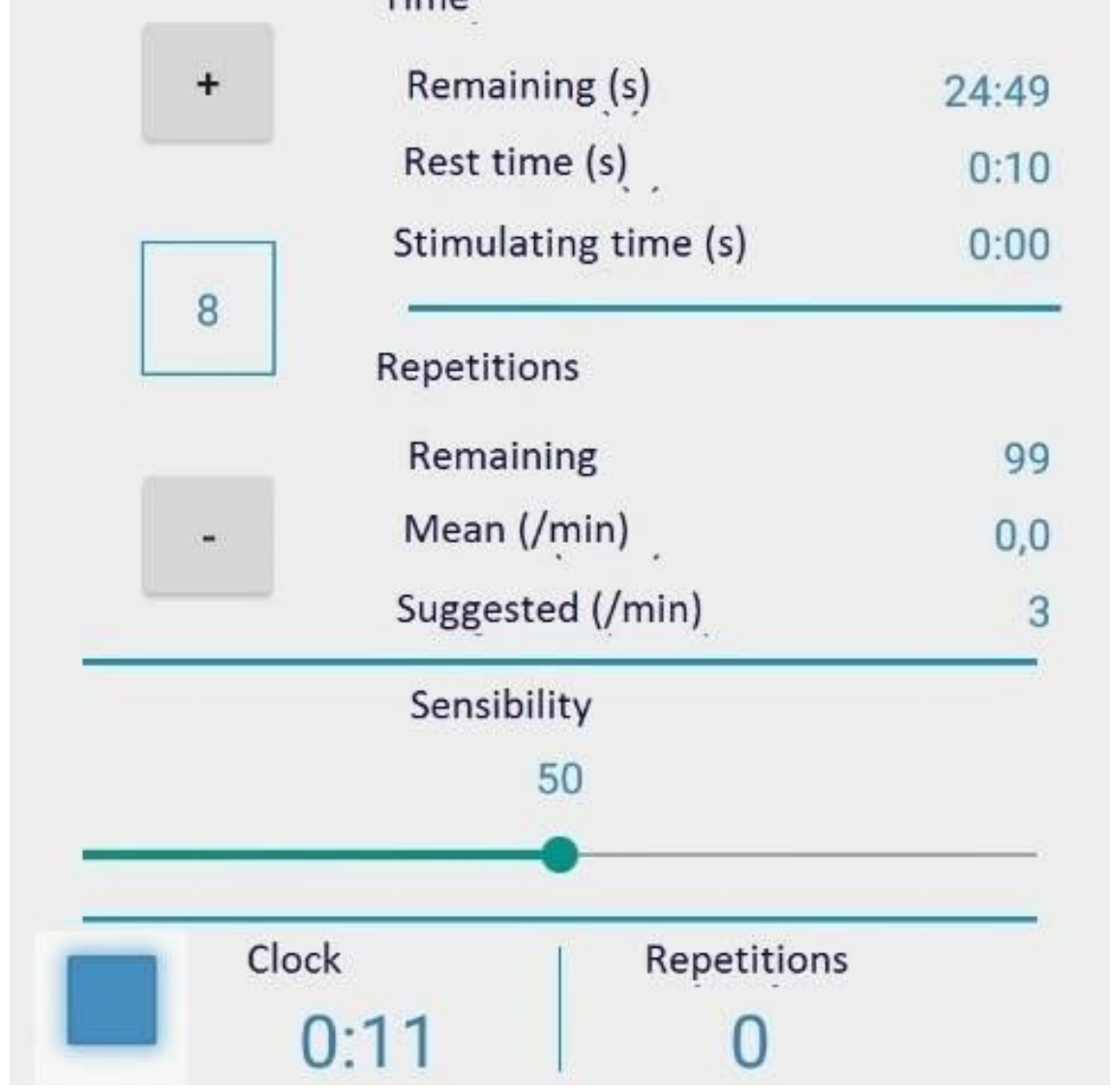

Time

\section{Figure 2}

Smartphone application procedure page, showing live information from the current session to the patient. 


\section{Neuro Stim Web App}

\section{Procedure Report}

\section{Procedure ID: 99}

Mode: Functional

Start Date: 31-01-2018

End Date: 31-01-2018

start Time: 11:19:42

End Time: 11:37:23

Session Duration: 17:41

Reos. Executed: 40

Stimulating Time: $7(\mathrm{~min}) \mathbf{4 4}(\mathrm{sec})$

Resting Time: 9 ( $\mathrm{min}$ ) 57(sec)

\section{EMG Data}

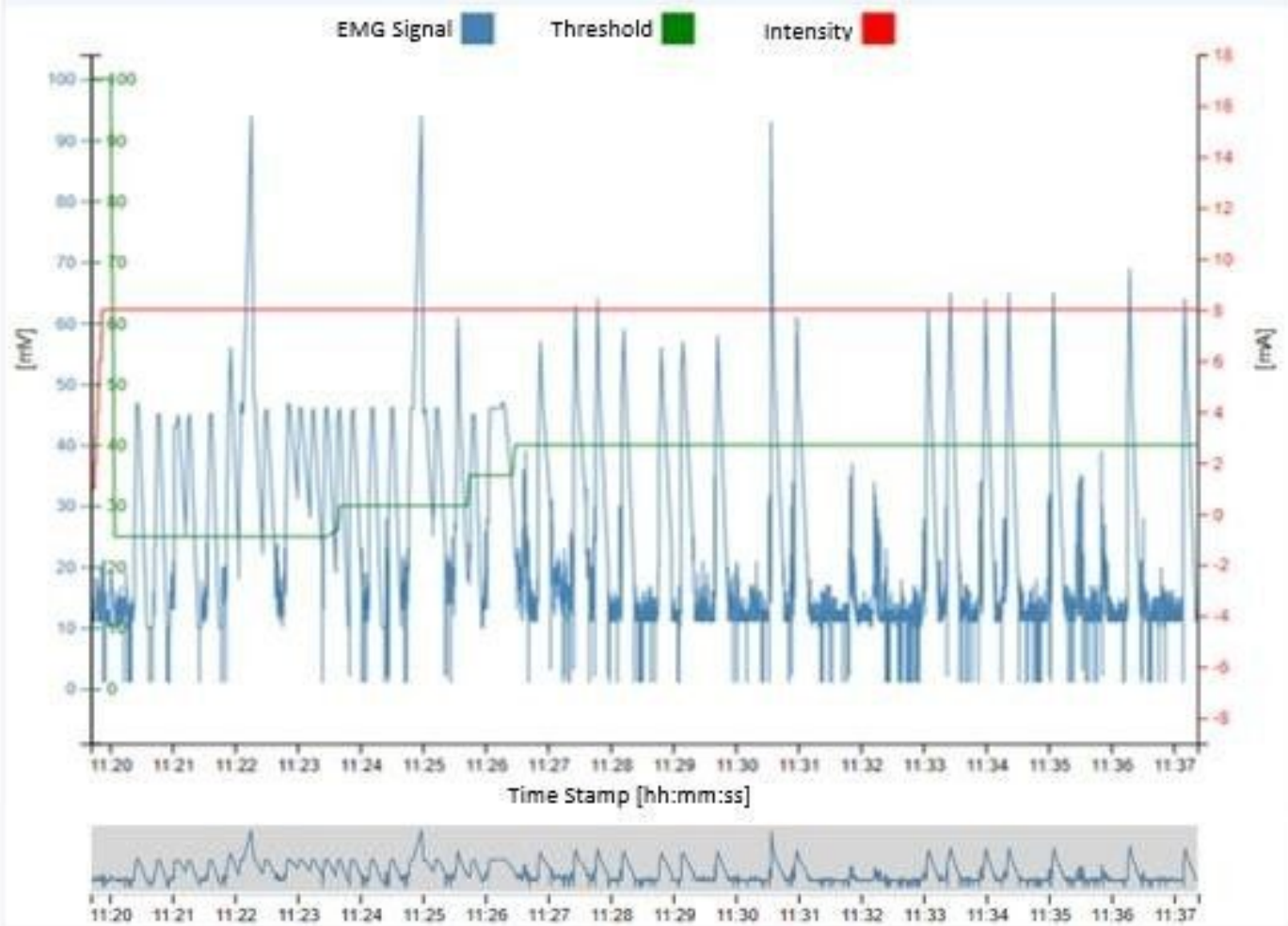

요 ashboard

A Print

(Q) Navigation

- 'Back

힐 Logout

\section{Figure 3}

The web interface dashboard shows the usage of data collected by the Smartphone application and sent to the server remotely through the internet. 